\title{
Multiple irregularities and thrombus in a patient with COVID-19 presenting with ST-segment elevation myocardial infarction: a case
}

\section{report}

\author{
Bhupesh Shah ${ }^{1 *}$, Harshal Shah', Darshil Shah ${ }^{2}$
}

\begin{abstract}
Background: Multiple thrombi are likely to develop in Coronavirus disease 2019 (COVID-19) patients. Hence, achieving successful outcomes following catheterization becomes quite challenging in such patients.

Case presentation: A 36-year-old female presented with ST-segment elevation myocardial infarction (STEMI). Coronary angiography revealed multiple irregularities in the coronary tree as well as thrombi. Although computed tomography imaging of the thorax was normal, reverse transcription-polymerase chain reaction (RT-PCR) confirmed the diagnosis of COVID-19. The outcome was good TIMI flow after a successful primary angioplasty in acute myocardial infarction (PAMI), and the patient was isolated and then switched to oral anticoagulants (clopidogrel) for dual antiplatelet therapy (DAPT) therapy.

Conclusion: This case emphasizes the management of a COVID-19 patient for PAMI.

Keywords: ST-Segment Elevation Myocardial Infarction, Coronary Angiography, Coronavirus Disease 2019, Multiple Thrombi, Primary Angioplasty in Acute Myocardial Infarction, India
\end{abstract}

\section{Background}

On March 11, 2020, World Health Organization (WHO) proclaimed the novel coronavirus disease (COVID-19) outbreak a global pandemic [1]. Although respiratory tract infections are typical symptoms and signs of COVID-19, cardiovascular manifestations including microvascular and macrovascular thrombi most frequently appear in COVID-19 patients. These thrombi can lead to multi-organ system injury, and failure [2], hence timely detection and prompt management are of utmost importance. Here, we reported a unique ST-segment elevation myocardial infarction (STEMI) case that abruptly manifested into multiple thrombi due to COVID-19.

\section{Case Report}

A 36-year-old female presented to the Emergency Department of HCG Hospitals, Ahmedabad- Gujarat, India, on 8 May 2021 with a three-day history of chest pain. The patient had an unremarkable medical history. On arrival, her blood pressure was $120 / 80 \mathrm{mmHg}$, and her pulse rate was over 100 beats per

*Correspondence: shahbhupesh@hotmail.com

${ }^{1 *}$ Department of Cardiology, HCG Hospitals, Ahmedabad-380006, Gujarat, India.

Full list of author information is available at the end of the article minute. Laboratory values signified low lymphocyte count and elevated High-Sensitivity Cardiac Troponin-I (hscTn-I). Echocardiography revealed an absence of Regional Wall Motion Abnormality (RWMA). The patient had unexplained tachycardia without pulmonary congestion and an S3 gallop. Coronary angiography displayed a mild diffuse lesion in the Right Coronary Artery (RCA), Left Circumflex (LCX) lesion step down in the reference diameter of the main vessel artery after proximal LCX, and Left Anterior Descending Artery (LAD) - First Diagonal Artery (D1) bifurcation lesion with medina class $(1,1,1)$. It further revealed multiple irregularities in the coronary tree with thrombus (Fig 1).

It was planned to perform PAMI of the LAD artery. On account of low lymphocyte count, multiple vessel segments were harboring thrombus-like lesions and unexplained tachycardia without pulmonary congestion and an S3 gallop. We considered the possibility of COVID-19. The patient was advised for Real-Time Reverse Transcriptase Polymerase Chain Reaction (RT-PCR) for Severe Acute Respiratory Syndrome Coronavirus-2 (SARS-CoV-2). The result of RT-PCR was positive; however, computed tomography imaging of the thorax was normal. In light of RT-PCR findings, a definitive diagnosis of COVID-19 was made. Under stringent precautions, the 
PAMI was started. LAD was pre-dilated with a short-length balloon. Then, a 3 x $32 \mathrm{~mm}$ drug-eluting stent (DES) stent was implanted, followed by post-dilation with a short-length balloon. The final angiography report showed impressive outcomes in terms of good TIMI flow.
After the PAMI procedure, the patient was isolated and then switched to clopidogrel (novel oral anticoagulants) from ticagrelor/prasugrel for Dual Antiplatelet Therapy (DAPT) therapy.

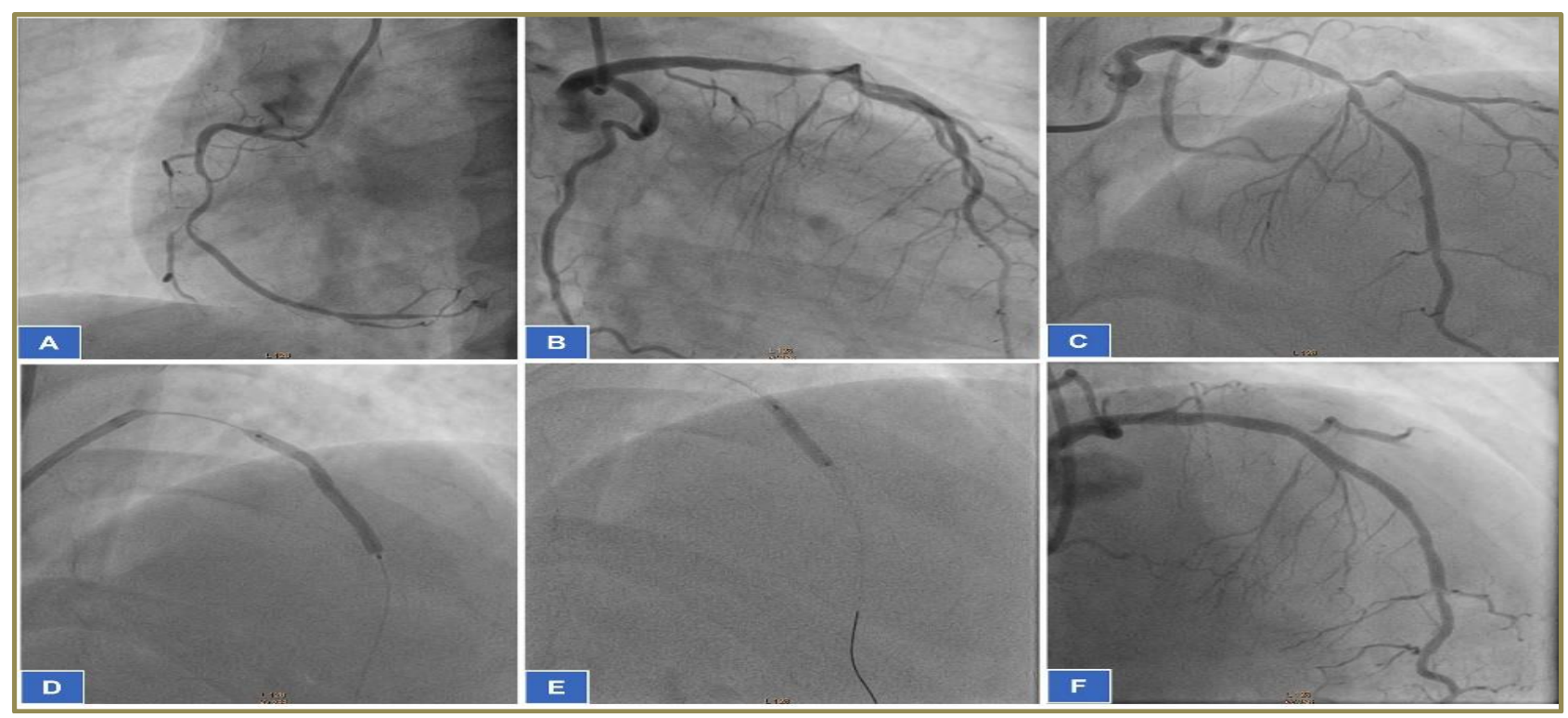

Fig 1. (a) Coronary angiography showing mild diffused lesion in the right coronary artery, (b) Coronary angiography showing left circumflex lesion to step down in the reference diameter of the main vessel artery after proximal LCX, (c) Coronary angiography showing LAD-D1 bifurcation lesion with medina class (1,1,1), (d) Implantation of a 3 x $32 \mathrm{~mm}$ DES following pre-dilation of LAD with a short length balloon, (e) post-dilation of the stent with a short length balloon.

\section{Discussion}

In this case, low lymphocyte count, multiple vessel segments harboring thrombus-like lesions, and unexplained tachycardia without pulmonary congestion and S3 gallop raised doubt for COVID-19. Since the outbreak of COVID-19 across the world, numerous studies have demonstrated a correlation between the severity of COVID-19 and lymphopenia, a condition described by abnormally low counts of lymphocytes [3-5]. At present, the molecular mechanisms of COVID-19 related thrombi have gained more attention; nevertheless, the exact mechanism behind it is still obscure. It is assumed to be an elevated level of pro-inflammatory cytokines [6], which activate the coagulation cascade and inhibit fibrinolysis. A procoagulant state in COVID-19 is preliminarily caused by Tumor Necrosis FactorAlpha (TNF- $\alpha$ ), Interferon-Gamma (IFN- $\gamma$ ), and Interleukin-1 (IL-1). This procoagulant state can result in migration and adhesion of leukocytes, activation and adhesion of platelet, and endothelial dysfunction, which leads to thrombus formation [7]. Numerous studies have recently revealed elevated thrombus formation in COVID-19 patients without previous cardiac diseases [8, 9]. Choudry et al. [7] described an exciting finding of higher thrombus burden in patients presenting with STEMI and concomitant COVID-19 infection. To the best of our knowledge, the present case is the second reported case of multiple irregularities and thrombus in the coronary tree of a STEMI patient with COVID-19 infection simultaneously. Effect of COVID-19 on STEMI patients could be either direct with a high risk of developing acute heart failure or indirect via the elevated delay between first medical contact and primary percutaneous coronary intervention on account of the recent contamination measures for COVID-19 that are followed by tertiary-care centers [10]. Nevertheless, it is assumed that the worst clinical scenario could be a combination of COVID-19 and STEMI in the same patient. However, we achieved promising outcomes in such a complex case. We recommend using a potent new generation of P2Y12 inhibitors such as prasugrel to prevent acute stent thrombosis and achieve promising outcomes following PAMI. In light of the ongoing COVID-19 pandemic, as per our recommendation, more emphasis should be given to maximize the protection of healthcare personnel as well as the hospital environment during any catheterization procedure. We strictly/strongly recommend adherence to guidelines that have been released by the Joint venture of The Society for Cardiovascular Angiography and Interventions (SCAI), the American College of Cardiology (ACC), and the American College of Emergency Physicians (ACEP) for the care of patients with STEMI during the COVID-19 pandemic [11]. The present case brought to light the probability of delaying door-to-Balloon (D2B) time due to cautious management of COVID-19 patients. Such a dealy can be avoided by competent staff as well as timely pre-operative planning. Our motto to inspire other physicians and achieve success is "not spend time but invest it."

\section{Conclusion}

This case highlights the effectiveness of PAMI as the standard of care for patients suffering from STEMI and COVID-19 simultaneously. The authors emphasize strict adherence to COVID-19 guidelines during catheterization procedures as per expert consensus. 
Abbreviation

STEMI: ST-Segment Elevation Myocardial Infarction; PAMI: Primary Angioplasty in Acute Myocardial Infarction; TIMI: Thrombolysis in Myocardial Infarction; WHO: World Health Organization; COVID-19: Novel Coronavirus Disease; hscTn-I: High-Sensitivity Cardiac Troponin-I; RWMA: Regional Wall Motion Abnormality; RCA: Right Coronary Artery; LCX: Left Circumflex; D1: First Diagonal Artery; RT-PCR: Real-Time Reverse Transcriptase Polymerase Chain Reaction; SARS-CoV-2: Severe Acute Respiratory Syndrome Coronavirus-2; TNF- $\alpha$ : Tumor Necrosis Factor-Alpha; IFN- $\gamma$ : Interferon-Gamma; IL-1: Interleukin-1; SCAI: Society for Cardiovascular Angiography and Interventions; ACC: American College of Cardiology; ACEP: American College of Emergency Physicians; D2B: Door-to-Balloon.

\section{Declaration}

Acknowledgment

None.

\section{Funding}

The authors received no financial support for their research, authorship, and/or publication of this article.

Availability of data and materials

Data will be available by emailing shahbhupesh@hotmail.com.

\section{Authors' contributions}

BP was the principal investigator of this manuscript and approved the final manuscript. BP, $\mathrm{HS}$, and $\mathrm{DH}$ were responsible for the study concept, design, writing, reviewing, and editing of the manuscript in its final form.

\section{Ethics approval and consent to participate}

We conducted the research following the Declaration of Helsinki. Ethical permission was granted by [HCG Hospitals, Ahmedabad380006, India, 2021]. The participant consent form was secured. All Coronary angiography images (in Figure 1) presented in the current study belong to NHL Municipal Medical College, Ahmedabad, Gujarat, India.

Consent for publication

Not applicable

Competing interest

The authors declare that they have no competing interests.

Open Access

This article is distributed under the terms of the Creative Commons $\begin{array}{llll}\text { Attribution } & 4.0 & \text { International License }\end{array}$ (http://creativecommons.org/licenses/by/4.0/), which permits unrestricted use, distribution, and reproduction in any medium, provided you give appropriate credit to the original author(s) and the source, provide a link to the Creative Commons license, and indicate if changes were made. The Creative Commons Public Domain Dedication waiver (http://creativecommons.org/publicdomain/zero/1.0/) applies to the data made available in this article, unless otherwise stated.
Author details

${ }^{1}$ Department of Cardiology, HCG Hospitals, Ahmedabad-380006, Gujarat, India. ${ }^{2}$ Department of Cardiology, NHL Municipal Medical College, Ahmedabad-380006, Gujarat, India.

Article Info

Received: 18 November 2021

Accepted: 21 December 2021

Published: 31 December 2021

References

1. World Health Organization WHO, director [report] [cited March 11, 2021]. Available from: https://www.who.int/directorgeneral/speeches/detail/who-director-general-s-opening-remarksat-the-media-briefing-on-covid-19---11-march-2020. Accessed on 25 July 2021.

2. Genovese L, Ruiz D, Tehrani B, Sinha S. Acute coronary thrombosis as a complication of COVID-19. BMJ Case Rep. 2021;14(3):e238218. https://10.1136/bcr-2020-238218

3. Tan L WQ, Zhang D, Huang Q, Tang Yi-Quan, Wang Q, Miao H. Correction: Lymphopenia predicts disease severity of COVID-19: A descriptive and predictive study. Sig Transduct Target Ther. 2020;5:33. https://10.1038/s41392-020-0159-1

4. Tavakolpour S, Rakhshandehroo T, Wei EX, Rashidian M Lymphopenia during the COVID-19 infection: what it shows and what can be learned. Immunol Lett. 2020;225:31-32. https://10.1016/j.imlet.2020.06.013

5. Wu C, Chen X, Cai Y, Xia J, Zhou X, Xu S, et al. Risk factors associated with acute respiratory distress syndrome and death in patients with coronavirus disease 2019 pneumonia in Wuhan, China. JAMA Intern Med. 2020;180(7):934-943. https://10.1001/jamainternmed.2020.0994

6. McGill SN, Ahmed NA, Christou NV. Endothelial cells: role in infection and inflammation. World J Surg. 1998;22(2):171-178. https://10.1007/s002689900366.

7. Choudry FA, Hamshere SM, Rathod KS, Akhtar MM, Archbold RA, Guttmann OP, et al. High thrombus burden in patients with COVID-19 presenting with ST-segment elevation myocardial infarction. J Am Coll Cardiol. 2020;76(10):1168-1176. https://10.1016/j.jacc.2020.07.022.

8. Bilaloglu S, Aphinyanaphongs Y, Jones S, Iturrate E, Hochman J, Berger JS. Thrombosis in hospitalized patients with COVID-19 in a New York City health system. JAMA. 2020;324(8):799-801. https://10.1001/jama.2020.13372.

9. Price LC, McCabe C, Garfield B, Wort SJ. Thrombosis and COVID-19 pneumonia: the clot thickens! Eur Respir J. 2020;56(1). https://10.1183/13993003.01608-2020

10. Tam C-CF, Cheung KS, Lam S, Wong A, Yung A, Sze M, et al. Impact of coronavirus disease 2019 (COVID-19) outbreak on STsegment-elevation myocardial infarction care in Hong Kong, China. Circ Cardiovasc Qual Outcomes;13(4):e006631. https://10.1161/CIRCOUTCOMES.120.006631.

11. Mahmud E, Dauerman HL, Welt FGP, Messenger JC, Rao SV, Grines C, et al. Management of acute myocardial infarction during the COVID-19 pandemic: a position statement from the Society for Cardiovascular Angiography and Interventions (SCAI), the American College of Cardiology (ACC), and the American College of Emergency Physicians (ACEP). J Am Coll Cardiol. 2020;76(11):1375-1384. https://10.1016/j.jacc.2020.04.03 